\title{
A simple plant mutation abets a predator-diversity cascade
}

\author{
Tobin D. Northfield ${ }^{1,3}$ William E. Snyder, ${ }^{1}$ Gretchen B. Snyder, ${ }^{1}$ and Sanford D. Eigenbrode ${ }^{2}$ \\ ${ }^{1}$ Department of Entomology, Washington State University, Pullman, Washington 99164 USA \\ ${ }^{2}$ Department of Plant, Soil and Entomological Sciences, University of Idaho, Moscow, Idaho 83844 USA
}

\begin{abstract}
Resource consumption often increases with greater consumer biodiversity. This could result either from complementarity among consumers or the inclusion of particular key species, and it is often difficult to differentiate between these two mechanisms. We exploited a simple plant mutation (reduced production of surface waxes) to alter foraging within a community of aphid predators, and thus perhaps shift the nature of resulting predator diversity effects. We found that greater predator species richness dramatically increased prey suppression and plant biomass only on mutant, reduced-wax pea plants (Pisum sativum). On pea plants from a sister line with wild type, waxier plant surfaces, predator species richness did not influence predators' impacts on herbivores or plants. Thus, a change in plant surface structure acted to turn on, or off, the cascading effects of predator diversity. Greater predator richness encouraged higher densities of true predators but did not lead to greater reproduction by a parasitoid, Aphidius ervi; fecundity of each natural enemy species was similar for the two plant types. Behavioral observations indicated that although $A$. ervi was less likely to forage within species-rich predator communities, low-wax plants mitigated this interference by encouraging generally greater $A$. ervi foraging and thus high rates of aphid dislodgement (aphids dropped from plants to escape $A$. ervi, but not the other predators). Thus, only species-rich, low-wax plants simultaneously encouraged strong species-specific effects of $A$. ervi, and strong complementarity among the other predator species. In summary, our study provides evidence that diversity effects in predator assemblages are sensitive to habitat characteristics. Further, we show that a simple plant morphological trait, controlled by a single gene mutation, can dramatically alter the cascading effects of predator species richness on herbivores and plants.
\end{abstract}

Key words: aphid predation; Aphidus ervi; biodiversity; complementarity; pea plants; Pisum sativum; predator; species identity; trophic cascade.

\section{INTRODUCTION}

Communities that include more species generally consume more resources (Hooper et al. 2005, Cardinale et al. 2006). This pattern could result from species using different, and complementary, portions of the total resource pool, which would represent an emergent outcome of greater species richness in the strictest sense (species complementarity; Loreau and Hector 2001). Alternatively, greater consumption with increasing diversity can result from the presence of particularly influential species within species-rich communities (species identity), such that greater consumption is only indirectly related to species richness (Huston 1997, Tilman et al. 1997, Loreau 1998). Successful management of communities to encourage greater functional biodiversity depends upon the ability to differentiate between these two mechanisms (Srivastava and Vellend 2005). For example, when species complementarity drives diversity effects, strategies must be designed to

Manuscript received 1 March 2011; revised 4 August 2011; accepted 8 August 2011. Corresponding Editor: K. F. Raffa.

${ }^{3}$ Present address: Department of Zoology, University of Wisconsin, 250 North Mills Street, Madison, Wisconsin 53706 USA. E-mail: tobin.northfield@email.wsu.edu influence multiple species to exploit their potential complementarity (Srivastava and Vellend 2005). In contrast, species-identity effects require targeted conservation of the single most effective species (Finke and Snyder 2010).

Ecologists have struggled to differentiate speciescomplementarity and species-identity mechanisms as the root causes of biodiversity effects, largely because breadth and intensity of resource use often are fixed species traits that cannot be manipulated experimentally (Loreau and Hector 2001, Naeem and Wright 2003, Finke and Snyder 2008). Species with plastic foraging behavior, however, can provide a route out of this conundrum. Differences in foraging behavior often translate into differences in specific resources used (i.e., the resource niche) (Chase and Leibold 2003). Consequently, experimentally altering foraging behavior can influence resource-niche differences among some species. When paired with an independent manipulation of species richness, such behavioral manipulations might in turn differentiate species-complementarity from species-identity effects (Finke and Snyder 2008, Barton and Schmitz 2009). One approach to manipulating the resource niche is to condition focal species to exploit different resources (e.g., Finke and Snyder 2008). 
Alternatively, foraging behavior can be manipulated indirectly by altering the environment so that resource partitioning among species is enhanced or diminished. For example, structurally more complex environments tend to reduce foraging overlap among species (e.g., Griffin et al. 2009). Because behavioral differences among species rather than species per se are manipulated, these approaches avoid the need to measure interspecific differences in particular traits with the assumption that they are tied to resource-use differences among species (Strong et al. 1979, Lewin 1983).

Here, we manipulated a plant trait known to influence predator foraging behavior, in order to also alter foraging-behavior differences among predator species. In turn, this allowed us to determine how these behavioral differences impacted the strength of trophic cascades exerted by single vs. multiple predator species. We used behavioral observations to elucidate specific behavioral mechanisms contributing to these effects.

\section{Methods}

Natural history

The experiments reported here were conducted within a community of predators attacking pea aphids, Acyrthosiphon pisum, on pea plants (Pisum sativum). Locally dominant predator species include the predatory bugs Geocoris bullatus and Nabis alternatus, the ladybeetles Hippodamia convergens and Coccinella septempunctata, and the parasitoid wasp Aphidius ervi (Chang et al. 2004a, $b$ ). These predators span a diverse range of potentially complementary hunting styles, including active hunters (Geocoris and the ladybeetles), a sit-andwait predator (Nabis), and an endoparasitoid (Aphidius). Furthermore, these species sometimes differ in where they forage on the plant, which also might contribute to their complementarity (Straub and Snyder 2008). Consistent with these putative functional differences, on plants other than peas combinations of these natural enemy species have been shown to strengthen aphid suppression compared to any single predator species foraging alone (Snyder et al. 2006, Northfield et al. 2010). Pea aphids often drop from plants to evade capture when predators approach, so that non-trophic predator effects could also influence trophic cascades (Losey and Denno 1998).

Our work takes advantage of two near-isogenic pea lines that differ in expression of the mutation wel (Marx 1969), which reduces wax crystals over the aerial plant surface (Eigenbrode et al. 1998a). Both lines were developed from accession W6-15368 ("Marx 406", USDA-ARS Western Regional Plant Introduction Station, Pullman, Washington, USA). The accession segregates for the waxy trait and allowed us to select two lines, 406G (reduced wax) and 406N (normal wax; see Plate 1). These lines have been used to examine the effect of waxes on insect predators (Eigenbrode et al. 1998b, White and Eigenbrode 2000, Rutledge et al. 2003, 2008), revealing that crystalline waxes alter predators' ability to attach to plant surfaces and thus their foraging patterns within the canopy.

\section{Experiments}

While our experiments differed in the scale at which they were conducted, they shared a common design. In all cases we conducted a factorial manipulation of peaplant line (normal or reduced-wax pea lines), fullycrossed with a manipulation of natural enemy species richness $(0,1$, or 4 natural enemy species present). Our natural enemies were drawn from the pool of five natural enemy species described above. The low speciesrichness treatment consisted of separate monocultures of each of the natural enemy species, while the species-rich treatment was composed of each unique draw of four species from our pool of five natural enemies (that is, each natural enemy species was deleted from one predator-polyculture composition). Thus, predator species composition was varied at both low and high levels of predator species richness, so that no single predator species (or species-rich composition) would disproportionately influence the comparison of low vs. high richness levels (Snyder et al. 2006). There has been much recent debate over the relative merits of additive vs. substitutive (replacement series) designs for the manipulation of predator biodiversity (e.g., Schmitz 2007, Byrnes and Stachowicz 2009). For our experiments, we chose to hold total predator density constant across species-richness treatments within a replacementseries design, such that density differences did not confound our comparison of species-poor vs. speciesrich treatments (Byrnes and Stachowicz 2009). In our study system, which included five predator species, an additive manipulation of predator richness would have been particularly difficult to achieve because of the large number of predator-species combinations that would need to be included, and undesirable because total predator densities would be far higher in diverse than single-species communities (Schmitz 2007). Therefore, while additive manipulations of predator richness are useful in many situations (e.g., Northfield et al. 2010), we instead followed a replacement-series design. Following manipulation of predator richness, we tracked plant and predator impacts on aphid densities and behavior, predator densities and behavior, and plant biomass.

Field experiment.- Our experimental units were $2 \times 2$ $\times 2 \mathrm{~m}$ field cages, covered with fine mesh and constructed as previously described (Snyder et al. 2006). Each cage enclosed $P$. sativum plants of either the parental or mutant low-wax lines, infested with $A$. pisum. On plants of each morphological type, we included two replicates of each of the five predator species alone, and two replicates of each of the five unique species-rich predator compositions. Together with four replicates on each plant type of a no-predator control, this yielded 48 cages in total ([5 predator species $\times 2$ replicates of each species in monoculture $=10]+[5$ 
unique draws of 4 predators from a pool of 5 species $\times 2$ replicates of each $=10]+4$ no-predator controls $=24 ; \times$ 2 plant wax types $=48$ replicates). We note that while each predator species composition was not heavily replicated, because statistical comparisons were made between the low- vs. high-diversity treatments, replication of diversity level was relatively substantial (10 replicates of each diversity level at each level of wax density). Our field-cage experiment was conducted at the Washington State University Tukey Horticulture Orchard in Pullman, Washington, USA, in June 2007.

Each cage was planted with 20 4-week-old $P$. sativum plants that had been started in a greenhouse (16:8 h photoperiod; $22^{\circ}: 16^{\circ} \mathrm{C}$ temperature regime, with these light and temperature regimes held constant using supplemental light and heating/cooling). Two days after transplanting (on 21 June 2007), each plant was infested with two adult pea aphids taken from a field colony. These aphids were allowed $24 \mathrm{~h}$ to assume feeding positions, after which predators were released according to treatment. All predators were collected from surrounding alfalfa (Medicago sativa) fields, and used within $24 \mathrm{~h}$ of being collected. Predators were held before release singly, in plastic vials with supplemental water at $10^{\circ} \mathrm{C}$ in the dark. Predators of all species were released simultaneously into each cage, only adult predators were initially released, and predators were released into all cages on the same day. Cages that received predators were stocked with a total of 12 predator individuals: single-predator-species treatments received 12 individuals of one species, whereas speciesrich predator treatments received three individual adults of each of four predator species. These densities are well within the range of typical open-field predator densities on peas in our region (Chang et al. 2004a). We counted aphids just before predator release, and then again 14 and 21 days later, by carefully examining each plant in each cage and recording all aphids encountered. A fourth aphid-sampling date was originally scheduled for day 31 of the experiment, but on that date we found that aphids had killed the plants in many cages (making an aphid count impossible), so we terminated the experiment by harvesting all plants in each cage. We then collected remaining predators during three 15-min searches of each cage (as described in Snyder et al. [2006]). Harvested plants were dried for 14 days in a drying oven at $60^{\circ} \mathrm{C}$ and then weighed.

Microcosm experiment.-Here, our arenas were 45-L (10-gallon) glass aquaria covered on the top with a mesh-screen lid, each housing four 2-week-old pea plants (in $500-\mathrm{mL}$ pots) of either the normal or reduced-wax lines. These arenas were housed within the same controlled-environment greenhouse where plants were reared, as described above. First, we released five pea aphids onto each plant (20 aphids per cage), and allowed $24 \mathrm{~h}$ for these aphids to assume feeding positions. We then counted the aphids, and released either four individuals of a single predator species, or one individual of each of four predator species, as described previously for the field experiment. This experiment was conducted as a series of three blocks, separate in time (blocks were initiated on 3 and 25 October 2007 and 10 July 2008). Within each block, and separately on plants of each wax-density line, each predator-species composition was replicated once; three no-predator controls were also included on plants of each type. This yielded a total of 78 experimental units across the entire experiment ([2 richness levels $\times 5$ species treatments $=10+3$ controls $] \times 2$ wax levels $\times 3$ blocks $=78$ ). The locations of predators on plants (top, bottom, or middle third of the plant) and the number of aphids displaced from plants (found elsewhere in the cage) were documented every $2-3$ hours during photophase. After $48 \mathrm{~h}$ of predator observation, final aphid densities were measured and the experiment was terminated. Scotophase observations during the first two temporal blocks revealed that predators were never active at night, and so this practice was stopped and nighttime data were not analyzed.

Additional experiments in greenhouse arenas were valuable for several reasons. First, it was not logistically possible to observe aphid and predator behavior in our large field experiment. Second, in the field experiment we found that plants from the two wax lines inherently differed in the number of aphids they could support under field conditions (Fig. 1A); this apparently occurs because the lower surface-wax density of the $406 \mathrm{G}$ line leads to greater water stress and reduced reflectance of incident radiation, leading to greater plant heat stress (Eglinton and Hamilton 1967). These differences in aphid densities complicated interpretation of wax effects on predator-prey interactions. Therefore, we conducted a follow-up experiment in smaller greenhouse arenas, where behavioral observations were more easily conducted and water-stress was presumably less of an issue. Microcosms reproduced field community structure by including multiple predators, herbivores, and plants.

\section{Data analysis}

For the field experiment, we analyzed aphid-density and plant-mass data using two-way ANOVA to evaluate the effects of plant line (normal, reduced wax), predator levels $(0,1$, or 4 species $)$, and their interactions. We also tested for chance, random variation in aphid reproduction among replicates that may have caused any initial, confounding differences among treatments. However, this random variation had no effect on final aphid counts $\left(F_{1,41}=0.23, P=0.637\right)$, nor did these initial counts differ between treatments (all $P>0.1$ ) so initial densities were not included in the final statistical model. Aphid-count data from the greenhouse experiment were treated identically, except that the model also included a temporal-blocking factor. Next, for each response variable we conducted three planned contrasts (Proc GLM SAS 9.1.3; SAS Institute 2003) on aphid density (field and greenhouse) and plant biomass (field only). 

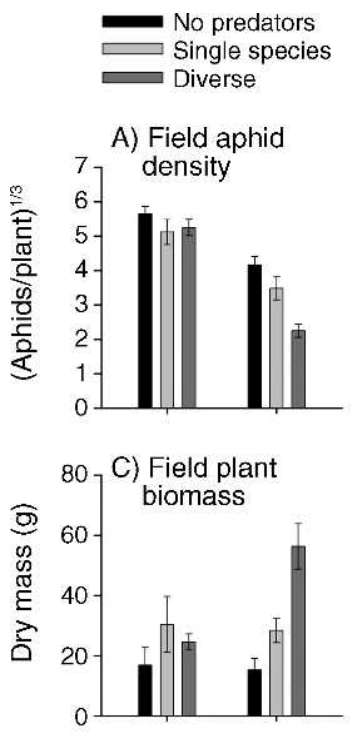

E) Greenhouse aphid

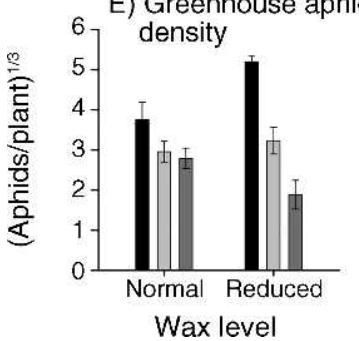

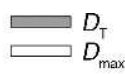

B) Field aphid
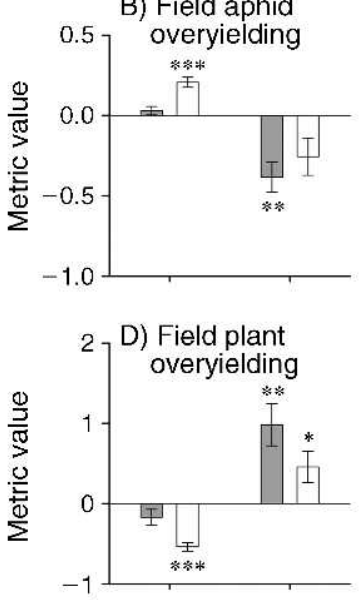

F) Greenhouse aphid

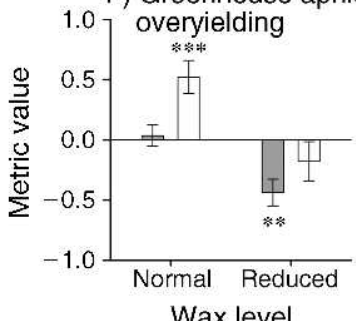

FIG. 1. (A) Mean densities of pea aphids in the field and (B) corresponding overyielding metrics; (C) final plant biomass from the field experiment and (D) corresponding overyielding metrics; and (E) final densities of pea aphids from the greenhouse experiment and $(\mathrm{F})$ corresponding overyielding metrics. Data are for three levels of predator manipulation $(0,1$, or 4 predator species, 4 being "diverse") fully crossed with two levels of plant wax level (normal or reduced) and are presented as means \pm SE. Overyielding metrics greater than (or less than) zero represent $(\mathrm{B}, \mathrm{F})$ aphid densities or $(\mathrm{D})$ plant biomass in the diverse predator treatments that are greater than (or less than) the best-performing single-species predator treatment $\left(D_{\max }\right)$ or the average of the single-species predator treatments $\left(D_{\mathrm{T}}\right)$. Asterisks denote metrics significantly different from 0 .

$* P<0.05 ; * * P<0.01 ; * * * P<0.001$.

The interaction term for the full ANOVA tests for effects of plant line on the magnitude of differences among the three predator treatments. Therefore, to compare only species-poor to species-rich predator treatments across plant lines we conducted an interaction contrast procedure. This test effectively removes the no-predator controls from the interaction term, while maintaining the error terms from the full experiment, and avoids problems associated with multiple tests (Bradu and Gabriel 1974). A second contrast isolated the overall effect of predators regardless of diversity level, to verify that predators were capable of significantly suppressing aphids on plants of both types; in this contrast we lumped the two predator-diversity levels together (see Snyder et al. 2006). Exploratory repeated- measures analysis revealed no significant treatment $x$ time interactions impacting aphid densities in the field experiment, and so aphid densities were analyzed as an average of the day 14 and day 21 counts. Final summed densities of generalist predators, and final parasitoid percentage parasitism were analyzed using a two-way ANOVA within the structure described above.

For both the field and greenhouse data we used several additional calculations to compare the relative degree of aphid suppression, and resulting cascading effects on plant biomass, exerted by single vs. multiple predator species. First, we calculated $D_{\mathrm{T}}$, the relative deviation of a species-rich treatment from the average performances of its constituent species when in monoculture (Loreau 1998). For the $i$ th species-rich treatment, the $D_{\mathrm{T}}$ value is calculated as $D_{\mathrm{T}, i}=\left(O_{i}-E_{i}\right) / E_{i}$, where $O_{i}$ is the observed number of aphids or plant biomass in the $i$ th species-rich treatment, and $E_{i}$ is the mean number of aphids or plant biomass of the singlespecies treatments included in the $i$ th species-rich treatment. Next, we calculated $D_{\max }$ to determine if the species-rich treatments performed better than the best single species, which is a more conservative test for emergent species richness effects (Loreau 1998). Here, for the $i$ th species-rich treatment $D_{\max , i}=\left(O_{i}-M_{\max }\right) /$ $M_{\max }$, where $M_{\max }$ is the value recorded for the "best performing" single-species treatment (i.e., the treatment with the lowest aphid density or greatest plant biomass) for a particular level of wax density. Because $D_{\max }$ values depend greatly on the values of a single monoculture treatment, one $A$. ervi single-species, reduced-wax replicate was removed from the calculation of $M_{\max }$, due to a lack of initial aphid establishment in that cage. We did not remove this treatment from the other analyses, because ANOVA is more robust to a single outlier than is $D_{\max }$. One-sample $t$ tests were conducted in SYSTAT (version 11; SPSS 2004), separately for each wax type, to determine if respective $D_{\mathrm{T}}$ and $D_{\max }$ means significantly differed from 0 .

To evaluate the behavioral observation data collected during the greenhouse study, we conducted two MANOVA's (Proc GLM SAS 9.1.3; SAS Institute 2003) to determine if predator foraging on different portions of plants (top, middle, and bottom third) was affected by the main and interactive effects of plant line, predator species richness, and predator identity. First, we used MANOVA to evaluate differences among all natural enemy species. In the second MANOVA we evaluated differences between parasitoids and true predators (all true predator species were lumped in this second analysis). Parasitoids were separated from true predators because we observed differences in foraging behavior between predators and the parasitoid consistent with earlier published work (e.g., Snyder and Ives 2001), and because preliminary data examination indicated no substantial differences among true-predator species in their responses (see also Snyder et al. 2006). We calculated the mean number of aphids 
displaced from plants (i.e., aphids found on the cage floor or walls) at each observation time using the factors A. ervi density, true-predator density, plant line (normal vs. reduced wax), predator species richness (single species vs. four species), and every potential interaction term, using weighted least-squares robust regression (Yohai 1987; Proc Robustreg SAS, SAS Institute 2003). There was no significant block effect $(P>0.05)$, and this factor was removed from the model.

All data were cube-root transformed prior to analysis to reduce skew (Chen and Deo 2004), except for aphid displacement data, which were overdispersed at the tails, rather than skewed (Appendix A). Overdispersion is common in predator behavioral data, and not accounting for such effects can lead to inflation of type 1 error (Link and Karanth 1994). Thus, we accounted for this lack of normality through weighted least-squares robust regression (Yohai 1987).

\section{RESULTS}

\section{Field experiment}

Both aphid densities and final plant biomass were significantly impacted by an interaction between predator species richness and plant line (aphid counts $F_{1,42}=$ $6.16, P=0.017$; Appendix B: Table B1; plant biomass $F_{1,42}=5.56, P=0.023$; Appendix B: Table B2): aphid densities were lower, and plants larger, when species-rich predator communities foraged on low-wax plants, but predator diversity had no effect on normal-wax plants (Fig. 1A, C). Thus, predator species richness strengthened trophic cascades only on low-wax, but not normal, plants. Overall aphid densities were significantly higher on normal than reduced-wax plants $\left(F_{1,42}=55.3, P<\right.$ $0.001)$, and predators significantly reduced aphid densities on plants of both types $\left(F_{1,42}=6.83, P=0.012\right)$.

Indeed, on low-wax plants the cascading effects of predator richness on the herbivore and plant both significantly exceeded what even the single most effective natural enemy species (the parasitoid Aphidus ervi) could achieve on its own: on this plant line the $D_{\max }$ values (a comparison of the diverse predator treatments to the best-performing single-species predator treatment) for aphid suppression were marginally significantly lower than 0 , and the $D_{\max }$ value for plant biomass was significantly higher than 0 (aphids $D_{\max }, t_{9}$ $=-2.20, P=0.055$, Fig. 1B; plants $D_{\max }, t_{9}=2.35, P=$ 0.044 , Fig. 1D). Thus, the diversity effects that we recorded could not be explained by the inclusion of particularly effective single-predator species within diverse-predator communities. In contrast, when diverse-predator communities foraged on normal-wax plants aphid suppression was significantly weaker, and plants were significantly smaller, than the single best predator species (on this plant line, the predator Geocoris bullatus) could achieve on its own (aphid $D_{\max }$, $t_{9}=6.75, P=0.001$; plant $D_{\max }, t_{9}=-10.51, P<0.001$; Fig. 1B, D). Rather, on normal-wax plants the performance of diverse predator assemblages could be

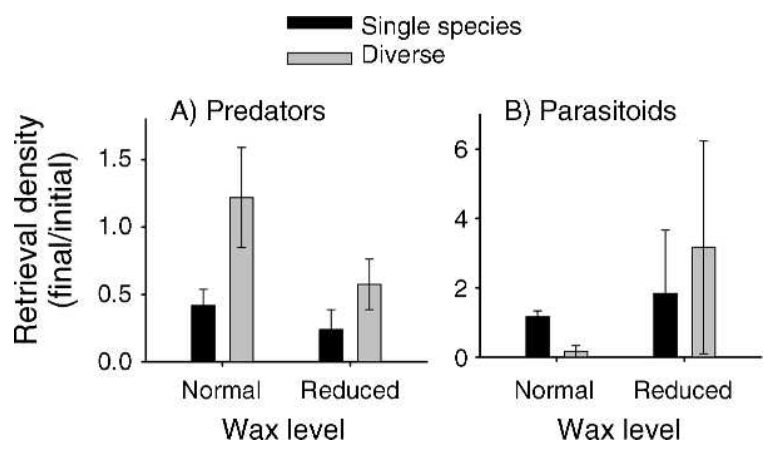

FIG. 2. (A) Predator and (B) parasitoid retrieval from the field experiment, by predator richness treatment and plant wax level (as in Fig. 1). Data are means \pm SE.

precisely predicted using the average performances of constituent predator species when in monoculture: (aphids $D_{T}, t_{9}=1.17, P=0.272$; plant biomass $D_{T}, t_{9}$ $=-1.71, P=0.122$; Fig. 1B, D). Thus, emergent predator species-richness effects occurred and were harmful to aphids and beneficial to plants on mutant low-wax plants, but predator-richness effects on normal-waxlevel plants were entirely neutral.

Final densities of true predators were significantly higher within species-rich than species-poor predator communities $\left(F_{1,32}=5.77, P=0.022\right.$; Fig. 2$)$, regardless of plant wax type $\left(F_{1,32}=0.03, P=0.873\right.$; Fig. 2$)$. Final percentage parasitism of aphids by $A$. ervi tended to be lower in species-rich than species-poor predator communities, although this trend was not statistically significant $\left(F_{1,16}=1.93, P=0.183\right.$; Fig. 2$)$. So, the four true predators clearly benefitted from being embedded within species-rich predator communities, whereas $A$. ervi did not (Fig. 2). Because of the difficulty of sexing many of these predator species and the large number of individuals that had to be handled, we were unable to sex predators before release to ensure a 50:50 sex ratio (except for $A$. ervi, where only mated females were released). However, because predator densities generally were higher in the diverse-predator communities where densities of each predator species were lowest, lack of available mates/reproductive females did not appear to entirely eliminate any benefits of greater species richness for predator densities.

\section{Microcosm experiment}

For aphid densities in the smaller-cage study, results were nearly identical to the field experiment: aphid densities were impacted by a strong interaction between plant-wax type and predator species richness, with diverse-predator communities significantly strengthening aphid suppression only on mutant, low-wax plants $\left(F_{1,60}=4.21, P=0.013\right.$; Fig. 1E; Appendix B: Table B3). Importantly, in the greenhouse overall aphid densities did not inherently differ between normal and reducedwax plants $\left(F_{1,60}=1.30, P=0.259\right.$; Fig. $1 \mathrm{E}$; Appendix B: Table B3), such that the interaction between plant type 


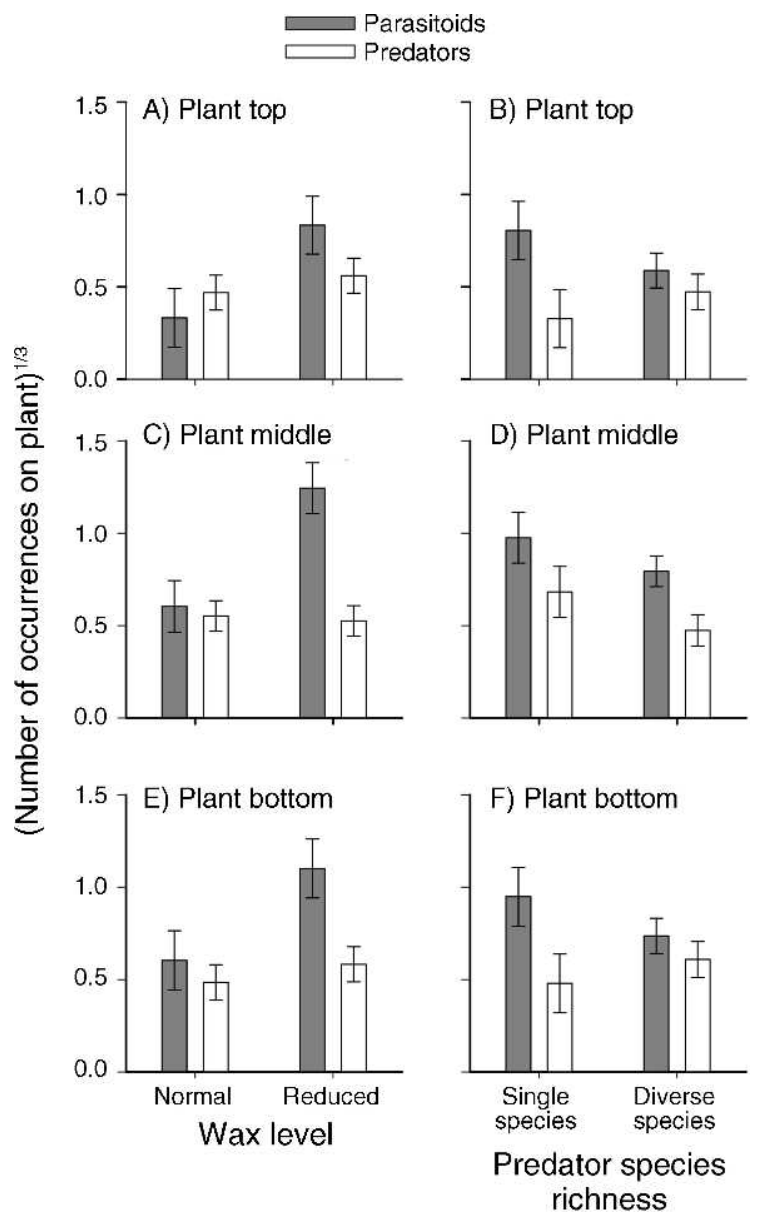

FIG. 3. Per capita number of times parasitoids (gray bars) and predators (white bars) were found on (A, B) the plant top third, $(\mathrm{C}, \mathrm{D})$ the middle third, and $(\mathrm{E}, \mathrm{F})$ the bottom third, sorted by (A, C, E) plant wax level or (B, D, F) predator species richness. Data are least-squares means $\pm \mathrm{SE}$. (The data were cube-root transformed to improve normality and are presented as transformed.)

and predator species richness could not have resulted from systematic differences in aphid densities on plants of the two types. Also as in the field experiment, $D_{\max }$ values for aphids were lower than 0 when diversepredator communities foraged on low-wax plants, although not significantly so, and $D_{\max }$ values were significantly greater than 0 when diverse-predator communities foraged on normal-wax plants $\left(t_{14}=\right.$ 3.806, $P=0.002$; Fig. 1F). Altogether, this experiment provides further support for the field observation that species-rich predator communities benefitted low-wax plants, but not normal-wax plants.

The smaller scale of this study also allowed us to make observations of predator and herbivore behavior, and we found widespread, although complex, effects of our treatments. When all predator species were compared, we found that neither plant line (predator species $\times$ plant line interaction: $F_{12,162}=1.12, P=0.349$;
Appendix C) nor predator-richness level (predator species $\times$ predator species richness interaction $F_{3,61}=$ $1.25, P=0.298)$ significantly affected where species foraged on plants. However, A. ervi was significantly more likely to be recorded on plants when they foraged among only conspecifics, rather than within a mix of conspecific and heterospecific predator species (predator type $\times$ predator species richness interaction: $F_{3,73}=3.14$, $P=0.030$; Fig. 3B, D, and F). Thus, predator diversity acted to reduce $A$. ervi occurrence on plants. However, on the reduced-wax plant line occurrence on plants increased significantly more for A. ervi than for the other predators, compared to when foraging on normal wax plants (predator type $\times$ plant line interaction: $F_{3,73}=$ 3.36, $P=0.023$; Fig. 3A, C, and E ). Thus, while the presence of other predators disrupt A. ervi foraging, this effect was rather ameliorated on reduced-wax plants by the overall higher wasp foraging rates that these plants engendered.

Only A. ervi regularly triggered aphid dropping behavior (A. ervi density: $\chi^{2}(1)=98.83, P<0.001$; Fig. 4), an effect that was particularly strong on reduced-wax plants $(A$. ervi density $\times$ plant line interaction: $\chi^{2}(1)=26.83, P<0.001$; Fig. 4; Appendix B: Table B4). Furthermore, aphid dropping in cages containing A. ervi scaled linearly with $A$. ervi occurrence on plants $\left(\chi^{2}(1)=9.50, P=0.002\right.$; Appendix B: Table B5). Wax level appeared to have no direct effect on aphid responses to $A$. ervi once differing plant-occurrence rates by wasps was accounted for $(A$. ervi visit $\times$ plant line interaction: $\left.\chi^{2}(1)=0.30, P=0.587\right)$,

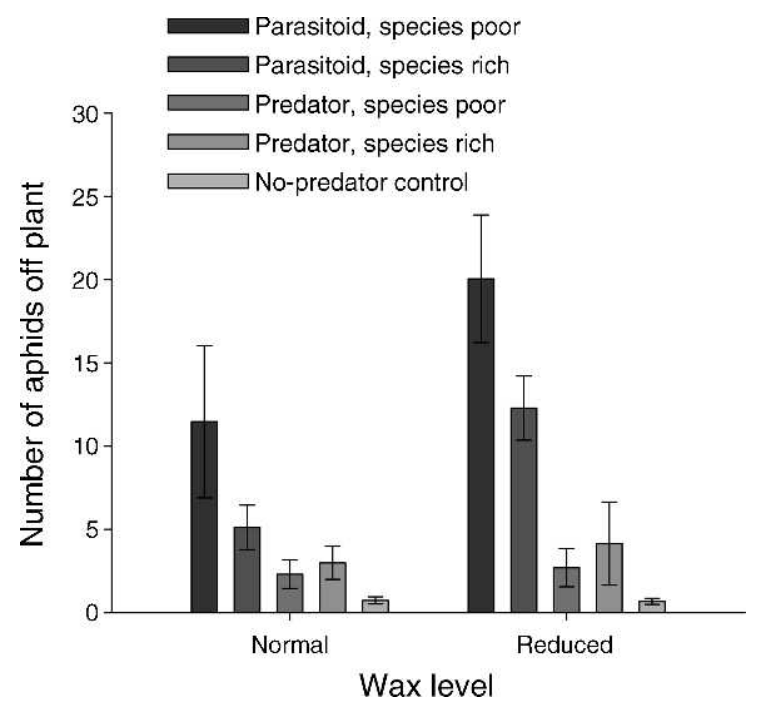

FIG. 4. The number of aphids displaced off plants in treatments containing either four Aphidus ervi (parasitoid, species poor), a single $A$. ervi and three true predators (parasitoid, species rich), four individuals of a single true predator species (predator, species poor), one individual of each of four different true predator species (predator, species rich), or no predators or parasitoids (no-predator control). Data are means $\pm \mathrm{SE}$. 


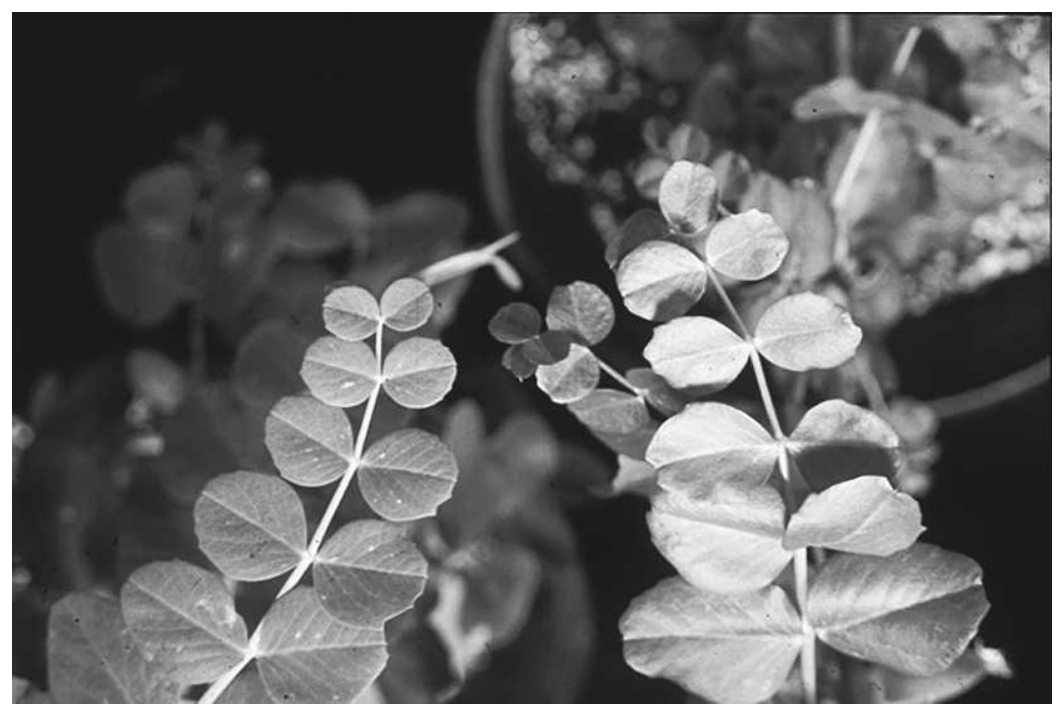

Plate 1. Pea lines used in the study. Line 406N (left) has a wild-type epicuticular wax bloom, and line 406G (right) has a reduced-wax phenotype caused by a mutation at locus WEL. Photo credit: S. D. Eigenbrode.

suggesting that $A$. ervi displaced more aphids on reduced-wax plants simply because wasps alighted upon these plants more often than normal-wax plants. In contrast to $A$. ervi, other predators did not have a significant role in aphid displacement (predator density $\left.\chi^{2}(1)=1.04, P=0.307\right)$ and did not influence aphid displacement by wasps $(A$. ervi density $\times$ predator density interaction $\left.\chi^{2}(1)=1.12, P=0.290\right)$.

\section{DiSCUSSION}

We found that the cascading effects of predators were influenced by a strong interaction between plant-surface morphology and predator species richness. On mutant reduced-wax plants in the field, species-rich predator communities killed more aphids, and thus promoted larger plants, than was seen across the single-predatorspecies communities (Fig. 1A, C). Indeed, on reducedwax plants species-rich predator communities initiated a significantly stronger trophic cascade than could even the single most effective natural enemy species, the parasitoid Aphidus ervi, when present alone (Fig. 1B, D). In stark contrast, on normal plants predator richness did not act to strengthen aphid suppression or increase plant biomass, and the performance of species-rich predator communities could be precisely predicted from the performances of each species when alone (e.g., multienemy effects were entirely additive; Fig. 1B, D). Thus, increasing predator richness was beneficial to mutant reduced-surface-wax pea plants, but not to normal plants. Our experimental design included all possible predator-community compositions within both speciespoor and species-rich treatments, and held total predator densities constant across species richness levels. Thus, neither the disproportionate representation of particular species or multi-species compositions, or differences in initial total natural-enemy densities, confounded our isolation of species richness effects (Snyder et al. 2006).

A possible complication when interpreting the results of our field-cage experiment is that aphid densities were significantly higher on normal than low-wax plants independent of any predator effects. Lower pea aphid densities are typical of the mutant peas under field conditions, likely reflecting the greater water stress these plants endure during hot sunny days (the surface wax layer serves to aid plants in water retention; Eglinton and Hamilton 1967). These inherent differences among pea lines raised the possibility that predator-richness effects were recorded on low-wax plants only because aphid densities were lower overall, so that it was easier to detect predator impacts of any type. However, several lines of reasoning argue against this explanation. First, in the field, predators significantly reduced aphid densities on plants of both types; it was only the low- and highrichness predator treatments that further separated on low-wax plants (Fig. 1A). Second, under greenhouse conditions overall aphid densities did not differ between the two types of peas (Fig. 1E), yet we again recorded increased prey consumption by species-rich predator communities on low-wax, but not normal-wax, pea lines (Fig. 1E, F).

Thus, by using otherwise nearly genetically identical pea lines differing in surface morphology, we were able to encourage or extinguish an impact of predator richness on aphids and plants. This provided an opportunity to search for differences in species densities and/or behavior across the two pea lines that could explain these differing trophic cascades, and we found that true predators and the parasitoid A. ervi clearly differed in their responses to both our plant-morphology and predator-richness manipulations. In the field experiment, final true-predator densities were significantly 
higher within species-rich than species-poor predator communities (Fig. 2A). The 31-day period of our experiment was sufficient to allow true predators to reproduce, and for young produced in situ to reach the final juvenile stages (e.g., Snyder et al. 2006), so these effects on predator density represented a combination of heightened survivorship of adults and juveniles and/or heightened reproduction by the adults initially released. However, the benefits of species richness for truepredator densities were not impacted by plant-wax type, consistent with our failure to find foraging-behavior differences between reduced-wax and normal plants during our behavioral observations. Therefore, predator-density effects alone could not explain the strong interaction between predator species richness and plant type that strengthened our trophic cascades.

The impacts of our treatments on A. ervi densities and foraging behavior were both more widespread, and more complex, than those observed for other predators. In the field-cage experiment, final wasp-recovery rates were no different in the presence of other predators compared to when A. ervi foraged alone (Fig. 2B). This suggested that, unlike true predators, $A$. ervi did not benefit from being within species-rich communities. Similarly, in our behavioral observations we saw reduced occurrence on plants when $A$. ervi foraged in species-rich communities, compared to when they foraged only with conspecifics (Fig. 3). This is consistent with other studies showing that parasitoids avoid sites where generalist predators have previously foraged, apparently to lessen their offspring's risk of falling victim to intraguild predation (e.g., Nakashima et al. 2004). Also unlike the other predator species, which showed no differences in time spent on the two pea lines, A. ervi spent significantly more time on reduced-wax peas. This is consistent with earlier work showing that $A$. ervi forage more on reduced-wax pea plants, apparently by spending less time in grooming behaviors to remove plant wax accumulated on their tarsi (Chang et al. 2004b). Thus, altogether, increasing predator species richness and reducing plant-wax density had opposing, but additive, impacts on $A$. ervi foraging behavior: $A$. ervi occurred on plants less often in speciesrich communities, but this effect was ameliorated by the greater overall wasp foraging on low-wax plants.

Pea aphids drop from plants when disturbed by natural enemies, a non-trophic effect that can strongly impact pea aphid feeding rates, densities, and impacts on plants (Losey and Denno 1998, Nelson et al. 2004). We found that the parasitoid $A$. ervi was far more likely to trigger aphid dropping behavior than any of the other predator species (Fig. 4). This non-trophic effect was diminished when $A$. ervi foraged within species-rich communities because, overall, wasps were less likely to occur on plants housing heterospecifics (Fig. 3). However, because low-wax plants encouraged greater overall time spent on plants by $A$. ervi, these plants encouraged relatively robust rates of aphid dislodgement by wasps even in the presence of other predators (Appendix B: Table B4). Aphid dropping behavior increased with the number of times $A$. ervi alighted upon plants, and this per visit dropping rate was not influenced by plant type (Appendix B: Table B5). Thus, $A$. ervi served a unique functional role by being particularly likely to incite aphid antipredator behavior, which reduces aphid feeding opportunities (Nelson et al. 2004) and heightens aphid exposure to ground-active predators (Losey and Denno 1998).

Altogether, our experiments suggest that only lowwax plants encouraged a predator-diversity cascade, because only these plants combined strong predatorpredator complementarity with strong species-specific effects of $A$. ervi. Most predators benefited from foraging within species-rich communities, but this effect occurred on plants of both morphological types. Predator diversity reduced $A$. ervi foraging (and thus the wasp's likelihood of triggering aphid-dropping behavior), but low-wax plants offset this harm by encouraging generally greater $A$. ervi foraging. In summary, only when species-rich predator communities foraged on low-wax plants did we record the merging of high predator densities due to predator-predator complementarity with strong trophic and non-trophic impacts particular to the wasp. Therefore, our present study illustrates the sensitivity of predator-diversity effects to habitat conditions, including those that can vary among host plants due to simple genetic variation.

A limitation of our study is that we considered just one plant species, which was developed for agricultural use. Thus, it remains unclear how broadly our results apply to other plant species embedded within agricultural or natural communities, including more species at each trophic level. However, we note that there often is considerable intraspecific variation in surface-wax density in naturally occurring plants, with some individuals displaying substantially higher or lower wax density than the species mean (Müller and Riederer 2005), suggesting that the type of surface-wax mediated effects that we recorded on peas could occur elsewhere. As a further caveat, because our two plant lines differed in surface-wax density, it is possible that other plant characteristics were also indirectly impacted. For example, the production or release of plant semiochemicals, often used by insect predators to locate their herbivore prey, might have also been impacted by differing surface chemistry (Desneaux and RamirezRomero 2009). Further work will be needed to examine these other, indirect effects of surface-wax alteration.

Recently, ecologists have struggled to disentangle the relative importance of strong effects of particular species, vs. complementary differences among species, as drivers of biodiversity effects (Huston 1997, Tilman et al. 1997). Most biodiversity-ecosystem function experiments use a statistical approach to partition the two, attributing any variance that cannot be explained by species-identity effects to interspecific complementity 
(Loreau 1998, Loreau and Hector 2001). With this approach the degree of complementarity is inferred, rather than directly demonstrated, and it often is unclear whether all or just some species make unique contributions to the process(es) of interest. More recently an experimental approach has been deployed, wherein communities are manipulated to (directly or indirectly) heighten or lessen species-specific differences in resource use (e.g., Finke and Snyder 2008, Barton and Schmitz 2009, Griffin et al. 2009, Levine and HilleRisLambers 2009; our present study). This allows direct demonstration of complementarity's contribution to emergent diversity effects. Thus far, such studies have verified that improved community performance results from reduced resource competition (Finke and Snyder 2008, Griffin et al. 2009), reduced interference competition (Barton and Schmitz 2009), or a combination of these two underlying mechanisms (Levine and HilleRisLambers 2009; our present study). Thus, these studies are providing rigorous experimental support for the classic models that first suggested a link between relatively weak intraspecific competition and greater overall resource use by communities (e.g., MacArthur 1958, Hutchinson 1959). A remaining challenge is to determine whether these same differences among species do in fact, in turn, explain the co-existence of diverse communities of competitors as the early modeling work suggests (Finke and Snyder 2008, Levine and HilleRisLambers 2009).

\section{ACKNOWLEDGMENTS}

We thank G. Clevenger for help with the field experiments. J. Rosenheim, D. Crowder, and three anonymous reviewers provided helpful comments on an earlier version of this paper. This project was supported by the National Research Initiative of the USDA Cooperative Research, Education and Extension Service (CSREES), grants number 2004-01215 and number 2007-02244.

\section{Literature Cited}

Barton, B. T., and O. J. Schmitz. 2009. Experimental warming transforms multiple predator effects in a grassland food web. Ecology Letters 12:1317-1325.

Bradu, D., and K. R. Gabriel. 1974. Simultaneous statistical inference on interactions in two-way analysis of variance. Journal of the American Statistical Association 69:428-436.

Byrnes, J. E., and J. J. Stachowicz. 2009. The consequences of consumer diversity loss: different answers from different experimental designs. Ecology 90:2879-2888.

Cardinale, B. J., D. S. Srivastava, J. E. Duffy, J. P. Wright, A. L. Downing, M. Sankaran, and C. Jouseau. 2006. Effects of biodiversity on the functioning of trophic groups and ecosystems. Nature 443:989-992.

Chang, G. C., J. Neufeld, D. Durr, P. S. Duetting, and S. D. Eigenbrode. 2004a. Waxy bloom in peas influences the performance and behavior of Aphidius ervi, a parasitoid of the pea aphid. Entomologia Experimentalis et Applicata 110:257-265.

Chang, G. C., C. E. Rutledge, R. C. Biggam, and S. D. Eigenbrode. 2004b. Arthropod diversity in peas with normal or reduced waxy bloom. Journal of Insect Science 4:18.

Chase, J. M., and M. A. Leibold. 2003. Ecological niches: linking classical and contemporary approaches. University of Chicago Press, Chicago, Illinois, USA.
Chen, W. W., and R. S. Deo. 2004. Power transformations to induce normality and their applications. Journal of the Royal Statistical Society Series B-Statistical Methodology 66:117130.

Desneaux, N., and R. Ramirez-Romero. 2009. Plant characteristics mediated by growing conditions can impact parasitoid's ability to attack host aphids in winter canola. Journal of Pest Science 82:335-342.

Eglinton, G., and R. J. Hamilton. 1967. Leaf epicuticular waxes. Science 156:1322-1335.

Eigenbrode, S. D., C. White, M. Rohde, and C. J. Simon. 1998a. Epicuticular wax phenotype of the wel mutation and its effect on pea aphid populations in the greenhouse and in the field. Pisum Genetics 29:13-17.

Eigenbrode, S. D., C. White, M. Rhode, and C. J. Simon. 1998b. Behavior and effectiveness of adult Hippodamia convergens (Coleoptera: Coccinellidae) as a predator of Acyrthosiphon pisum (Homoptera: Aphididae) on a wax mutant of Pisum sativum. Environmental Entomology 27:902-909.

Finke, D. L., and W. E. Snyder. 2008. Niche partitioning increases resource exploitation by diverse communities. Science 321:1488-1490.

Finke, D. L., and W. E. Snyder. 2010. Conserving the benefits of predator biodiversity. Biological Conservation 143:22602269.

Griffin, J. N., S. R. Jenkins, L. Gamfeldt, D. Jones, S. J. Hawkins, and R. C. Thompson. 2009. Spatial heterogeneity increases the importance of species richness for an ecosystem process. Oikos 118:1335-1342.

Hooper, D. U., et al. 2005. Effects of biodiversity on ecosystem functioning: a consensus of current knowledge. Ecological Monographs 75:3-35.

Huston, M. A. 1997. Hidden treatments in ecological experiments: re-evaluating the ecosystem function of biodiversity. Oecologia 110:449-460.

Hutchinson, G. E. 1959. Homage to Santa Rosalia, or why are there so kinds of many animals? American Naturalist 93:145159.

Levine, J. M., and J. HilleRisLambers. 2009. The importance of niches for the maintenance of species diversity. Nature 461:254-U130.

Lewin, R. 1983. Santa Rosalia was a goat. Science 221:636-639.

Link, W. A., and K. U. Karanth. 1994. Correcting for overdispersion in tests of prey selectivity. Ecology 75:24562459.

Loreau, M. 1998. Separating sampling and other effects in biodiversity experiments. Oikos 82:600-602.

Loreau, M., and A. Hector. 2001. Partitioning selection and complementarity in biodiversity experiments. Nature 412:7276.

Losey, J. E., and R. F. Denno. 1998. Positive predatorpredator interactions: enhanced predation rates and synergistic suppression of aphid populations. Ecology 79:21432152.

MacArthur, R. 1958. Population ecology of some warblers of northeastern coniferous forests. Ecology 39:599-619.

Marx, G. A. 1969. Two additional genes conditioning wax formation. Pisum Newsletter 1:10-11.

Müller, C., and M. Riederer. Plant surface properties in chemical ecology. Journal of Chemical Ecology 31:26212651.

Naeem, S., and J. P. Wright. 2003. Disentangling biodiversity effects on ecosystem functioning: deriving solutions to a seemingly insurmountable problem. Ecology Letters 6:567579 .

Nakashima, Y., M. A. Birkett, B. J. Pye, J. A. Pickett, and W. Powell. 2004. The role of semiochemicals in the avoidance of the seven-spot ladybird, Coccinella septempunctata, by the aphid parasitoid, Aphidius ervi. Journal of Chemical Ecology 30:1103-1116. 
Nelson, E. H., C. E. Matthews, and J. A. Rosenheim. 2004 Predators reduce prey population growth by inducing changes in prey behavior. Ecology 85:1853-1858.

Northfield, T. D., G. B. Snyder, A. R. Ives, and W. E. Snyder. 2010. Niche saturation reveals resource partitioning among consumers. Ecology Letters 13:338-348.

Rutledge, C. E., S. D. Eigenbrode, and H. Ding. 2008. A plant surface mutation mediates predator interference among ladybird larvae. Ecological Entomology 33:464-472.

Rutledge, C. E., A. Robinson, and S. D. Eigenbrode. 2003. Effects of a simple plant morphological mutation on the arthropod community and the impacts of predators on a principal insect herbivore. Oecologia 135:39-50.

SAS Institute. 2003. SAS version 9.1.3. SAS Institute, Cary, North Carolina, USA.

Schmitz, O. J. 2007. Predator diversity and trophic interactions. Ecology 88:2415-2426.

Snyder, W. E., and A. R. Ives. 2001. Generalist predators disrupt biological control by a specialist parasitoid. Ecology 82:705-716.

Snyder, W. E., G. B. Snyder, D. L. Finke, and C. S. Straub. 2006. Predator biodiversity strengthens herbivore suppression. Ecology Letters 9:789-796.
SPSS. 2004. SYSTAT, version 11. SPSS, Chicago, Illinois, USA.

Srivastava, D. S., and M. Vellend. 2005. Biodiversity-ecosystem function research: Is it relevant to conservation? Annual Review of Ecology Evolution and Systematics 36:267-294.

Straub, C. S., and W. E. Snyder. 2008. Increasing enemy biodiversity strengthens herbivore suppression on two plant species. Ecology 89:1605-1615.

Strong, D. R., L. A. Szyska, and D. S. Simberloff. 1979. Test of community-wide character displacement against null hypotheses. Evolution 33:897-913.

Tilman, D., C. L. Lehman, and K. T. Thomson. 1997. Plant diversity and ecosystem productivity: Theoretical considerations. Proceedings of the National Academy of Sciences USA 94:1857-1861.

White, C., and S. D. Eigenbrode. 2000. Leaf surface waxbloom in Pisum sativum influences predation and intra-guild interactions involving two predator species. Oecologia $124: 252-259$

Yohai, V. J. 1987. High breakdown-point and high-efficiency robust estimates for regression. Annals of Statistics 15:642656.

\section{Supplemental Material}

\section{Appendix A}

A figure depicting a normal quantile-quantile plot for aphid displacement data in greenhouse-microcosm arenas (Ecological Archives E093-038-A1).

\section{Appendix B}

Five ANOVA tables for statistical analysis (Ecological Archives E093-038-A2).

\section{Appendix C}

A figure depicting predator foraging locations in the greenhouse-microcosm arenas (Ecological Archives E093-038-A3). 